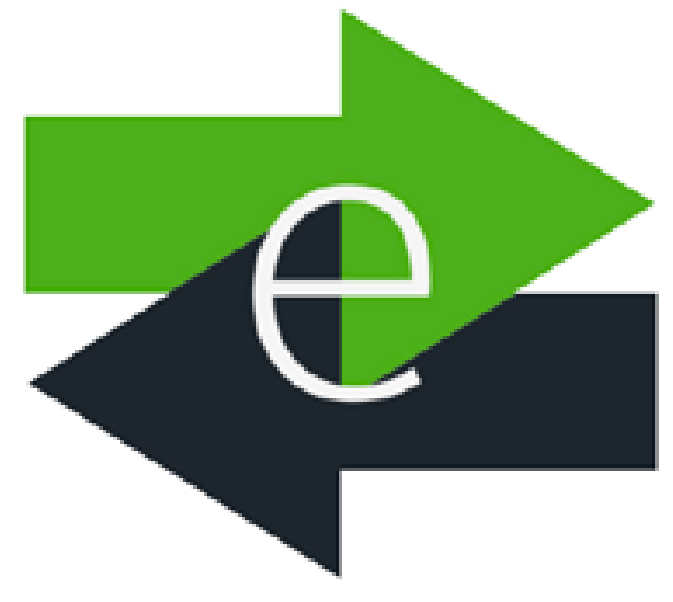

FIGURAS REVISTA ACADÉMICA DE INVESTIGACIÓN

ISSN 2683-2917

Vol. 2, núm. 2, marzo-junio 2021

https://doi.org/10.22201/fesa.figuras.2021.2.2

Esta obra está bajo una licencia

Creative Commons Atribución-NoComercial-

Compartirlgual 4.0 Internacional

\section{eLaboraHD: un proyecto pedagógico en humanidades digitales}

\author{
eLaboraHD: a Pedagogical Project \\ in Digital Humanities \\ https://doi.org/10.22201/fesa.figuras.2021.2.2.146
}

Ernesto Priani-Saisó

Univesidad Nacional Autónoma de México.

Facultad de Filosofía y Letras

eLaboraHD es un proyecto de pedagogía digital nacido de la necesidad de enseñar a estudiantes de nivel superior el uso y la aplicación de las herramientas digitales. Con esa base el proyecto ha crecido para impartir talleres a profesores e involucrar a quienes hacen herramientas y proyectos digitales orientados a la enseñanza en el impulso de adopción de estas herramientas.

\section{eLaboraHD}

A pesar de que muchas de las herramientas digitales con las que contamos hoy tienen ya algunas décadas, su aplicación en la enseñanza y la investigación en el campo de las humanidades -más allá del uso del procesador de palabras y las hojas de cálculo- ha tardado más de lo esperado.

Esto se puede explicar en buena medida por la resistencia, a menudo expresada como sospecha, de las humanidades por las innovaciones tecnológicas; pero muy particularmente por las digitales que comenzaron a invadir y a transformar los objetos de estudio privilegiados por las disciplinas humanísticas: el texto, la imagen y el archivo.

Sin embargo, a todo le llega su hora. La creciente digitalización de archivos, bibliotecas y museos ha hecho que, no sin cierta reluctancia, los humanistas comiencen a voltear -finalmente- hacia las herramientas digitales y perciban la necesidad de formarse en el uso de ellas y en el sentido de su aplicación en investigaciones humanísticas.

El proyecto eLaboraHD (https://elaborahd.unam. $\mathrm{mx} /$ ) comenzó en el año 2015 como un esfuerzo por contribuir a la formación en el uso de las herramientas y metodologías digitales de los estudiantes de las licenciaturas en humanidades de la Facultad de Filosofía y Letras de la UNAM. Cuando nace, las responsables del proyecto, Dra. Adriana Álvarez Sánchez y Dra. Miriam Peña Pimentel, impartían - de manera conjunta - un curso de metodología para la carrera de Historia: el Seminario Taller Especializado " $\mathrm{Hu}-$ manidades Digitales e Historia".

Ambas humanistas digitales habían percibido la necesidad de respaldar su curso con una investigación que les permitiera comprender, sistematizar y ampliar la enseñanza del uso de estas herramientas dado el interés y el impacto que el curso había tenido. Para ello solicitaron financiamiento al Programa de Apoyo a Proyectos para la Innovación y Mejoramiento de la Enseñanza (PAPIME) para el proyecto "Las 
Humanidades Digitales: un campo innovador para la enseñanza de la Historia", que fue el inicio formal de eLaboraHD. Desde entonces han recibido financiamiento para dos etapas más del proyecto, 2016-2018 y una más que inició en el 2019.

En un primer momento, el objetivo del proyecto fue tener un sitio web que sirviera de respaldo y guía a la impartición del taller, pero que a la vez fuera una referencia para la comunidad universitaria interesada en conocer las herramientas, su posible aplicación y su uso. Muy pronto el proyecto pasó de centrarse en la enseñanza para estudiantes de licenciatura, a extenderse a la impartición de talleres para profesores a través del Programa de Actualización y Superación Docente (PASD) de la UNAM, mientras se profundizaba en el conocimiento de la pedagogía de las humanidades digitales.

En su etapa más reciente, el proyecto se ha propuesto colaborar directamente con los proyectos digitales que desarrollan los profesores de la Facultad de Filosofía y Letras. El objetivo en este caso es visibilizar el trabajo docente digital, las situaciones que se enfrentan al carecer de un departamento especializado para este tipo de proyectos y las formas en que hemos resuelto esta problemática.

Como puede verse, el proyecto ha ido extendiéndose para cubrir a la mayoría de los actores que son relevantes para la enseñanza del uso de las herramientas digitales desde la perspectiva de las humanidades digitales: estudiantes, profesores y profesores/ investigadores. Queda pendiente, sin embargo, involucrar a administrativos y directivos que son también sujetos importantes.

En la actualidad, además de llevar a cabo un programa de identificación de proyectos digitales en humanidades y reuniones con sus responsables, el proyecto eLaboraHD acaba de lanzar un sitio web renovado (https://elaborahd.unam.mx/) concentrado en ofrecer lecciones, recursos y notas, además de la información general sobre el proyecto, a toda la comunidad universitaria y, de manera más amplia, a todo aquel interesado en el conocimiento, uso y aplicación de las herramientas.

Para ello, han hecho una primera selección de herramientas y las han convertido en breves lecciones didácticas sobre su uso y aplicación. Entre éstas encontramos lecciones sobre los gestores bibliográficos como Zotero, el uso de mapas digitales, la utilización de museos digitales y las propias bibliotecas digitales de la UNAM.

En el rubro de recursos, que son entradas para presentarlos y ligarlos con alguna de las lecciones, encontramos museos como el Metropolitan Museum of Art y una biblioteca como The Internet Archive, donde se explora qué ofrecen y por qué pueden ser de interés como fuentes para el trabajo académico.

Entre las notas encontramos la convocatoria a algunos eventos, apuntes sobre temas como el "Vocabulario digital" o la "Historia del arte y los museos digitales", en las que se ahonda sobre algún aspecto útil para quien se inicia en el uso de herramientas digitales para el estudio y la investigación.

El sitio también ofrece acceso a los resultados de la investigación, una encuesta y diversos artículos publicados, que permiten adentrarse aun más en el trabajo del grupo de investigación y, en general, en la pedagogía digital.

eLaboraHD dista mucho de haber concluido. Más bien, está comenzando a consolidarse como un proyecto de gran alcance dentro de la Universidad y un referente nacional y latinoamericano en materia de pedagogía digital. El trabajo por delante es amplio y puede ser muy rico porque, sin duda, se trata de un proyecto que se ocupa de un tema que ya en este momento es muy relevante, pero que tras la pandemia se ha vuelto fundamental y urgente. -

\section{Referencia}

eLaboraHD. 2019. Breves lecciones didácticas sobre su uso y aplicación. Entre éstas encontramos el uso de mapas digitales, la utilización de museos digitales y las propias bibliotecas digitales de la UNAM, en: https://elaborahd.unam.mx/ 